\title{
Guest editorial for special issue on modeling and analysis of human-machine systems in transportation
}

\author{
Jianhua Zhang1 - Tamsyn E. Edwards ${ }^{2}$
}

Published online: 8 November 2017

(C) Springer-Verlag London Ltd., part of Springer Nature 2017

The human has an important role in safety and efficiency in most safety-critical environments. Although some safetycritical domains have developed safety measures through automated systems or physical defenses (i.e., physical shutdown in the nuclear industry), environments especially within the transport domain are still reliant on the maintenance of human performance to sustain safety. For example, the air traffic controllers (ATCOs) are responsible for the safety and efficiency of all air traffic. As there are no physical measures that protect aircraft in flight, it is essential that controllers maintain a consistently high level of performance in order to maintain flight safety. Similar challenges also exist in the rail and road domains.

Safety and efficiency in transportation are dependent upon the human-machine system (HMS): the human operator, the technology, and the effective interaction between human and machine. Technology must not only perform effectively, efficiently and reliably, but also be designed to be usable and acceptable to the human operator. The human operator must be able to maintain a high level of performance in order to guarantee safety. The interaction between these components must be effective for the optimal operation of a transportation system. Each component plus the interaction between

Jianhua Zhang

zhangjh@ecust.edu.cn

Tamsyn E. Edwards

tamsyn.e.edwards@nasa.gov

1 School of Information Science and Engineering, East China University of Science and Technology, 200237 Shanghai, China

2 Airspace Operations Laboratory, Human Systems Integration Division, NASA Ames Research Center/San Jose State University, Moffett Field, CA, USA them can affect the overall performance and safety of the transportation system.

It is in this spirit that we, as the Guest Editors, are honored to have been given the opportunity to organize this Special Issue (SI) on 'Modeling and Analysis of HumanMachine Systems in Transportation' for Cognition, Technology and Work (CTW) journal. All the selected 11 papers represent the latest innovative research work in the areas of modeling, quantitative and qualitative data analysis, pattern recognition, and machine learning and deep learning, all of which are applied to safety-critical environments in the transportation domain. The organization of the SI was suggested and encouraged by Professor F. VANDERHAEGEN (University of Valenciennes, FRANCE), one of the EiCs of the CTW journal.

In the context of the ongoing rapid development in automation systems and technologies that is changing both the work environment and role of the human operators, research contributions in this SI considering the human operator's role in HMSs as well as issues of human-automation interaction and teaming are particularly timely and relevant. This SI delivers an overview of the essential research topics across transportation domains, and across multiple facets of the HMS. An overarching focus of this collection of 11 papers is the importance of the human operator as part of a whole HMS from the holistic standpoint. By combining this set of papers, this SI aims to provide a collection of innovative research work that deepens our understanding of how to enhance safety, efficiency and overall HMS performance in the field of transportation. The findings of each article have significant implications for supporting the human operator across several transportation domains, with specific implications and practical relevance for safety and efficiency.

The 11 papers contained in this SI provide novel perspectives and latest research work with applications to four 
transport domains-rail (Dadashi, Golightly and Sharples; Brandenburger and Jipp), road (Friedland, Snycerski, Palmer and Laraway), aviation (Borst, Bijsterbosch, Paassenn and Mulder; Edwards, Homola, Mercer and Claudatos; Nixon and Charles), and maritime (Patriarca and Bergstrom). In addition, issues related to diverse aspects of the HMS are explored in the remaining papers, from human-centric design considerations of operator decision support (Borst, Bijsterbosch, Paassenn and Mulder; Dadashi, Golightly and Sharples; Friedland, Snycerski, Palmer and Laraway), to the model-based assessment of operator cognition and mental workload (MWL) (Edwards, Homola, Mercer and Claudatos; Nixon and Charles; Yin, Liu, Liu, Zhang and Wang; Zhang, Wang and $\mathrm{Li}$ ) and factors that influence operator performance (Brandenburger and Jipp).

Therefore, this SI provides the unique contribution of presenting the state-of-the-art research results across transportation domains, facilitating exchanges of new research ideas and methodology and comprehensive comparisons of different techniques for modeling and analysis of HMSs in different transportation domains, as well as exploring a breadth of issues that are primary theoretical or practical concerns in HMSs. The papers can be categorized into the following three main themes:

1. Human-centric design and tools that support the human operator Borst, Bijsterbosch, Paassenn and Mulder consider the ecological interface design that can support ATCOs' fault diagnosis of an automated decision aid system. Surrounding this theme, Dadashi, Golightly and Sharples examine whether or not providing more information to human operators leads to better operational decisions in a rail operation task, with important implications for future tool design. Furthermore, Friedland, Snycerski, Palmer and Laraway explore the supportive tool of glare-reducing glasses for drivers, with clear links to supportive design as well as implications for driving safety.

2. Modeling, analysis and evaluation of the cognitive variables of the human operator In a series of three papers, Zhang and his Intelligent Systems Group apply advanced machine learning techniques (including transfer learning, deep learning, and imbalanced data classification) to electrophysiological time-series data based pattern classification of MWL of operators, whereas Nixon and Charles utilize wearable technology for electrophysiological measurement of ATCO state (including MWL) based on the notion of the human performance envelope. Yin, Liu, Liu, Zhang and Wang explore a unique neurophysiological method for measuring operator's affective state. Edwards, Homola, Mercer and Claudatos study the human performance envelope by exploring subjective measures of MWL and situational awareness in ATCOs, as well as the composite effect of these variables on operator performance. Their work is also linked to the third theme of the SI as follows.

3. Factor analysis of human operator performance Brandenburger and Jipp present their investigation into the effects of automatic speed control and expertize on train driver performance degradation. Additionally, Patriarca and Bergstrom present a novel application of FRAM to gain insight into the complexity of tasks carried out by operators in the maritime domain.

We believe that the present contributions provide state-ofthe-art research as well as some promising research avenues for future work in HMS modeling and analysis techniques in transportation. We hope that this issue, while not providing all the answers to the challenges in HMSs in transportation domain, could provide some solutions and identify open problems or new challenges for researchers and practitioners alike in the HMS field. The key message that we wish to convey through this Editorial is that we need to work together to establish a rapprochement between human and complex technical systems in many real-world safety-critical contexts including transportation field.

Finally, we wish to thank all the contributors to this SI for their effort in preparing and revising their manuscripts and the reviewers for carefully and critically evaluating the contributions for two or three rounds to maintain the scientific standard and reputation of the journal. 